\title{
799 NEOANTIGEN-CYTOKINE-CHEMOKINE MULTIFUNC- TIONAL NATURAL KILLER CELL ENGAGER FOR IMMUNOTHERAPY OF SOLID TUMORS
}

Xue Yao*, Sandro Matosevic. Purdue University, Lafayette, IN, United States

Background The effectiveness of natural killer (NK) cell-based immunotherapy against solid tumors is limited by the lack of specific antigens and the immunosuppressive tumor microenvironment (TME). Glioblastoma multiforme (GBM) is one such heavily immunosuppressive tumor that has been particularly hard to target and remains without a viable treatment. The development of novel approaches to enhance the efficacy of NK cells against GBM is urgently needed. NK cell engagers (NKCE) have been developed to enhance the efficacy of NK cell therapy.

Methods To improve the clinical efficacy of NK cell therapy, we are developing a new generation of multi-specific killer engagers, which consists of a neoantigen-targeting moiety, together with cytokine and chemokine-producing domains. Neoantigens are new antigens formed specifically in tumor cells due to genome mutations, making them highly specific tools to target tumor cells. Our engager has been designed to target Wilms' tumor-1 (WT-1), a highly specific antigen overexpressed in GBM among other solid tumors. This is done through the generation of an $\mathrm{scFv}$ specific targeting the complex of WT-1126-134/HLA-A*02:01 on the surface of GBM. On the NK cell side, the engager is designed to target the activating receptor NKp46. Incorporation of the cytokine IL15 within the engager supports the maturation, persistence, and expansion of NK cells in vivo while favoring their proliferation and survival in the tumor microenvironment. Additionally, our data indicated that the chemokine CXCL10 plays an important role in the infiltration of NK cells into GBM, however, GBM tumors produce low levels of this chemokine. Incorporation of a CXCL10-producing function into our engager supports intratumoral NK cell trafficking by promoting, through their synthetic production, increased levels of CXCL10 locally in the tumor microenvironment.

Results Collectively, this has resulted in a novel multifunctional NK cell engager, combining neoantigen-cytokine-chemokine elements fused to an activating domain-specific to NK cells, and we have investigated its ability to support and enhance NK cell-mediated cytotoxicity against solid tumors in vitro and in vivo against patient-derived GBM models. The multispecific engager shows both high tumor specificity, as well as the ability to overcome NK cell dysfunction encountered in the GBM TME.

Conclusions We hypothesize that taking advantage of our multi-functional engager, NK cells will exhibit superior ex vivo expansion, infiltration, and antitumor activity in the treatment of GBM and other solid tumors.

http://dx.doi.org/10.1136/jitc-2021-SITC2021.799 